\title{
Stephan Stein \& Sören Stumpf. 2019. Muster in Sprache und Kommunikation. Eine Einführung in Konzepte sprachlicher Vorgeformtheit (Grundlagen der Germanistik 63). Berlin: Erich Schmidt. 280 S.
}

Besprochen von Sarah Brommer: Leuphana Universität Lüneburg, Institut Deutsche Sprache und Literatur und ihre Didaktik, Universitätsallee 1, D-21335 Lüneburg, E-Mail: sarah.brommer@leuphana.de

https://doi.org/10.1515/zrs-2021-2063

„Das vorliegende Einführungsbuch basiert auf der Überzeugung, dass Idiomatizität, Formelhaftigkeit, Modellhaftigkeit, Vorgeformtheit usw. keine Randphänomene einer Sprache sind, sondern dass sprachliche Musterhaftigkeit vielmehr ein grundlegendes und überaus facettenreiches Wesenselement natürlicher Sprachen ist.“ - So die einleitenden Worte der Autoren Stephan Stein und Sören Stumpf. Dieser Überzeugung folgend ist es ihr Anliegen, „einen Überblick über die vorgeformten sprachlichen Phänomene der deutschen Gegenwartssprache zu geben" (S. 11) und das Konzept der Musterhaftigkeit gleichermaßen auf alle sprachlichen (Beschreibungs-)Ebenen zu beziehen, ,anhand authentischer Beispiele die jeweiligen Charakteristika sowie die kommunikative Relevanz von Musterhaftigkeit herauszuarbeiten" (ebd.) und mit ihrer Gesamtdarstellung eine Lücke im Bereich der Studienbücher zu schließen. Diesem Anspruch wird das Buch - soviel sei vorweggenommen - rundum gerecht.

Die Einführung zeichnet sich durch einen äußerst stringenten und schlüssigen Aufbau und ein hohes Maß an Leser`innenführung aus. Nach einem einleitenden Kapitel wird Musterhaftigkeit auf den einzelnen sprachlichen Ebenen vom Kleinen zum Großen: Wortebene, Mehrwortebene, Satzebene, Textebene, Gesprächsebene, Diskursebene - in den Blick genommen. Die darauffolgenden Kapitel beleuchten Musterhaftigkeit im Spracherwerb und in Bezug auf sprachliche Varietäten sowie die Perspektive der Sprachkritik. Alle Kapitel sind in sich abgeschlossen, auch unabhängig voneinander lesbar und folgen der gleichen Struktur. Zu Beginn jedes Kapitels werden zunächst als Einstieg Beispiele aus dem aktuellen Sprachgebrauch diskutiert, die zur Reflexion anregen und auf das jeweilige Kapitel einstimmen. Sodann folgt ein mit „Grundlagen“ überschriebenes Unterkapitel, das in die jeweils relevante Theorie oder Disziplin einführt (z.B. Kap. 5: Textebene $\Rightarrow$ Grundlagen: Textbegriff und Textualitätskriterien; Kap. 6: Gesprächsebene $\Rightarrow$ Grundlagen: Linguistische Gesprächsforschung; Kap. 8: Musterhaftigkeit im Spracherwerb $\Rightarrow>$ Grundlagen: Spracherwerb). Diese Grundlagenkapitel ermöglichen auch Leser^innen ohne größeres Vorwissen, die folgenden Darstellungen und Überlegungen nachvollziehen zu können. Am Ende 
jedes Kapitels wiederum folgen exemplarische Vertiefungen, die auf den Ausführungen aufbauen und diese anhand praktischer Beispiele weiterführen. Jedes Kapitel schließt mit einer kurzen Zusammenfassung und Literaturhinweisen, die kurz kommentiert sind.

Angesichts der inhaltlichen Breite ist es nicht verwunderlich, dass das Buch eine Gemeinschaftsarbeit ist (anders als es die bibliographischen Daten vermuten lassen, die Stein und Stumpf als alleinige Autoren ausweisen). Einzelne Kapitel wurden von Martin Wengeler (Diskursebene), Natalia Filatkina (Musterhaftigkeit im Spracherwerb) und Andrea Bachmann-Stein (Musterhaftigkeit und sprachliche Varietäten) sowie unter Mitarbeit von Carina Hoff (Gesprächsebene) verfasst. Dies findet leider nur im innenliegenden Titelblatt sowie im Vorwort Erwähnung, hätte aber auch durch Nennung im Inhaltsverzeichnis und bei den einzelnen Kapiteln gewürdigt werden können. Nun zum Inhalt im Einzelnen.

In Kapitel 1 („Muster in Sprache und Kommunikation: Begriffsbestimmung, theoretische Konzepte und Untersuchungsmethodik“) wird einerseits gefragt, was Musterhaftigkeit umfasst (extensionale Begriffsbestimmung, Kap. 1.1), andererseits, was ,sprachlich musterhaft' heißt (intensionale Begriffsbestimmung, Kap. 1.2). Mit ihrem Begriffsverständnis schließen die Autoren an den Forschungsdiskurs an (vgl. z.B. Bubenhofer 2009; Feilke 1996; Steyer 2004, 2013) und bestimmen sprachlich Musterhaftes durch „Rekurrenz, Konventionalität, Historizität und (Re-)Aktualisier- bzw. Reproduzierbarkeit einschließlich situativer Anpassbarkeit und individueller Veränder- und gesellschaftlicher Wandelbarkeit“ (S. 19). Damit fallen unter musterhaften Sprachgebrauch sowohl lexikalisch stark spezifizierte Einheiten mit niedrigerem Abstraktionsgrad (z.B. Phraseme oder Wortbildungsmuster) als auch lexikalisch kaum oder nicht spezifizierte Einheiten mit höherem Abstraktionsgrad (z.B. Argumentationsmuster oder Denkstereotype). Eine graphische Übersicht (S. 18) veranschaulicht das Spektrum anhand von Beispielen und macht auch deutlich, dass die sprachliche Ebene, auf der sich das Musterhafte verorten lässt (Wort, Satz, Text usw.), nicht mit dem Lexikalisierungs- oder Abstraktionsgrad korreliert. Formelhafte Texte (wie die Ansage im Zug) sind bspw. stärker lexikalisiert als Satzmuster mit bestimmten semantischen Rollen. Die Entscheidung für den Begriff (z. B. S. 25) bzw. das Konzept (z.B. S. 13) ,Musterhaftigkeit‘ (analog ,sprachlich Musterhaftes‘ und ,Muster') gegenüber anderen Begriffen/Konzepten wie Phraseologizität, Formel(haftigkeit), Modell(haftigkeit), Verfestigung, Routine, Schemata, Konstruktion und anderen mehr (Kap. 1.3) begründen die Autoren zum einen mit der Anschaulichkeit und Allgemeinverständlichkeit des Begriffs, zum anderen mit seiner Eignung, ,die auf ganz unterschiedlichen sprachlichen Ebenen angesiedelten [und unterschiedlich komplexen] Phänomene in ihrem gemeinsamen Kern zu erfassen“ (S. 25). Der weitere Vorteil, nämlich dass man mit der Wahl des Begriffs ,Musterhaftigkeit“ 
vermeidet, sich auf eine bestimmte Schule oder Denkrichtung festzulegen (wie bspw. bei ,Konstruktion“ oder ,Schema‘ der Fall), wird nur kurz gestreift, indem der Begriff als „theoretisch unvorbelastet“ (ebd.) charakterisiert wird. Daraufhin folgt die Anbindung von ,Musterhaftigkeit‘ an ausgewählte theoretische Konzepte (Kap. 1.4): an das Zentrum-Peripherie-Modell und den Prototypengedanken, an das Konzept der idiomatischen Prägung und das Ebenen-Modell (Feilke) sowie an die Konstruktionsgrammatik. Die Autoren geben dabei jeweils einen gut verständlichen Einblick in den aktuellen Forschungsstand, legen Stärken und Schwächen der Konzepte dar sowie Gemeinsamkeiten und Unterschiede zum Konzept der Musterhaftigkeit. Allein bei den Ausführungen zu Zentrum, Peripherie und Prototypizität irritiert, dass die Autoren zwar (zurecht) auf die Prager Schule verweisen, sich dann aber im weiteren Verlauf ausschließlich auf die Verwendung des Modells in der Phraseologie stützen; die Semantik bspw. bleibt unerwähnt, würde sich als theoretische Anbindung aber ebenso eignen (s. a. Mangasser-Wahl 2000). Dies scheint nicht ganz nachvollziehbar angesichts der Feststellung der Autoren, dass sich das breite Spektrum musterhaften Sprachgebrauchs eben nicht in den Gegenstandsbereich der Phraseologie zwängen lässt (vgl. S. 17). Es zeigt sich aber an verschiedenen Stellen des Buches, dass vor allem die Phraseologie wie auch die Konstruktionsgrammatik die hauptsächlichen theoretischen Bezugspunkte der Einführung bilden. Das abschließende Unterkapitel (Kap. 1.5) hebt schließlich die Relevanz, ja sogar Notwendigkeit von Korpusanalysen für die Untersuchung musterhaften Sprachgebrauchs hervor. Der Überblick über korpuslinguistische Verfahren ist anschaulich gestaltet und führt insbesondere in die Kookkurrenzanalyse als geeignete Untersuchungsmethode ein. Damit ist der theoretische und methodische Rahmen ausreichend abgesteckt und die einzelnen sprachlichen Beschreibungsebenen werden in den Blick genommen.

Muster auf Wortebene sind für Stein und Stumpf laut der Überschrift von Kap. 2 Wortbildungsmuster. Das mag auf den ersten Blick überraschen, denn auch der Gebrauch einzelner Wörter kann musterhaft sein; er ist es aber nur für eine bestimmte Textsorte oder Kommunikationssituation, und diese Musterhaftigkeit ist somit auf Text-, Gesprächs- oder Diskursebene zu verorten. Nach einer Einführung in die Wortbildung des Deutschen zeigen die Autoren auf, wie sich die Musterhaftigkeit bei der Bildung neuer Wörter sowohl auf struktureller als auch auf semantischer Ebene zeigt und dass es gleichzeitig Beschränkungen in der Wortbildung gibt, die ihrerseits ebenfalls musterhaft sind. Dabei erwähnen sie anhand von Beispielen auch Probleme bei der Modellierung. Dass auf den wichtigen Zusammenhang von Sprachsystem, Norm und Akzeptanz eingegangen und vorgeführt wird, „dass Wortbildungsprodukte existieren, die mustergerecht und systemkonform gebildet sind, trotzdem aber inakzeptabel erscheinen“ (S. 55) 
und der Norm zuwiderlaufen, ist besonders überzeugend und hätte auch in den weiteren Kapiteln stärker aufgegriffen werden können. Nach einem Abschnitt zur konstruktionsgrammatischen Perspektive auf Wortbildungsmuster folgen die exemplarischen Vertiefungen. Diese beziehen sich auf die Relevanz von Wortbildungsmustern im Rahmen des Fremdsprachenunterrichts sowie die Betrachtung von Wortbildung in der Jugendsprache. In diesem Punkt fällt die Darstellung meinem Empfinden nach zu vereinfachend aus und es hätte zumindest erwähnt werden müssen (das kann als wissenschaftlicher Konsens gelten), dass es die Jugendsprache als solche nicht gibt, sondern sich der Sprachgebrauch Jugendlicher höchst unterschiedlich gestaltet.

Einleitend zu Kap. 3 zur Musterhaftigkeit auf Mehrwortebene wird zurecht darauf hingewiesen, dass feste Mehrwortlexeme den „Untersuchungsgegenstand sprachlicher Vorgeformtheit par excellence“ (S. 67) bilden, dass aber gleichzeitig auch das in der Sprache vorhandene und im Sprachgebrauch genutzte Potential an Variation und Modifikation bei der Erforschung berücksichtigt und herkömmliche phraseologische Betrachtungsweisen überdacht werden müssen. Das Grundlagenkapitel rückt denn auch die kritische Betrachtung der Kriterien Polylexikalität, Festigkeit und Idiomatizität und die mit Schwierigkeiten verbundene Klassifikation von Phrasemen ins Zentrum. Im Folgenden wird die Musterhaftigkeit auf Mehrwortebene als Anlass genommen, (erneut) eine Brücke zu Phraseologie und Konstruktionsgrammatik zu schlagen. Unter Bezugnahme auf das Zentrum-Peripherie-Konzept werden schließlich Kollokationen als nicht oder nur schwach idiomatische Wortverbindungen sowie usuelle Wortverbindungen (im Text an anderer Stelle auch als usualisierte Mehrwortverbindungen bezeichnet) dem Grenzbereich der Phraseologie zugerechnet. Hier zeigt sich die Problematik, die im Kriterium der Festigkeit liegt und überleitet zum folgenden Kapitel, in dem es um den veränderten oder auch fehlerhaften Phrasemgebrauch geht. Das Vertiefungskapitel nimmt schließlich die Phraseodidaktik sowie Phraseme in der Kinder- und Jugendliteratur in den Blick.

Der Schwerpunkt des Kapitels 4 zur Musterhaftigkeit auf Satzebene liegt im Bereich valenzgrammatischer Überlegungen. Ausgangspunkt ist die Valenz des Verbs, die sich sowohl in der Anzahl als auch der Art der Ergänzungen sowie ihrer Semantik äußert und verschiedene vorgeformte Strukturgefüge, sog. Satzmuster (auch Satzmodelle oder Satzbaupläne), zur Folge hat. Die Beispiele führen vor Augen, dass das Musterhafte der syntaktischen Struktur ((morpho)syntaktische Muster) und der damit verbundenen semantischen Rollen (semantische Muster) bspw. im Vergleich zur Musterhaftigkeit auf Mehrwortebene weniger ins Auge fällt und der Abstraktionsgrad sehr viel höher ist. Relevant ist diese Art der Musterhaftigkeit insbesondere im Fremdsprachenunterricht, wo Valenzwörterbücher (hierzu eine der beiden exemplarischen Vertiefungen) hauptsächlich genutzt wer- 
den. Auch auf dieser Ebene stellen die Autoren den Bezug zur Konstruktionsgrammatik her, und sie sprechen sich explizit dafür aus, valenztheoretische und konstruktionsgrammatische Erkenntnisse zueinander in Beziehung zu setzen und die beiden Perspektiven integrativ zu behandeln.

Das Kapitel 5 zur Textebene thematisiert verschiedene Aspekte rund um Textsorten und Textmuster. Das Grundlagenkapitel fällt vergleichsweise ausführlich aus und geht bereits auf Textmustervariationen ein. Die Abgrenzung von ,Textsorte' als typologischem Begriff und ,Textmuster' als prozeduralem Wissen nach Heinemann \& Heinemann (2002) hätte aber geschärft werden können durch einen Bezug auf die Arbeiten von Ulla Fix (z.B. 1999), die mit ,Textsorte' die Tatsache erfasst, dass sich konkrete Textexemplare aufgrund ihrer gemeinsamen Merkmale zusammenfassen lassen, und mit ,Textmuster die dahinterstehende mentale Größe bezeichnet, die als Referenz dient. Im Folgenden wird einerseits die mikrostrukturelle Musterhaftigkeit auf Textebene (Formulierungsmuster), andererseits die makrostrukturelle Musterhaftigkeit (formelhafte Texte) betrachtet. Auch hier - wie in allen weiteren (Unter)Kapiteln - veranschaulichen gut gewählte Beispiele die verständlich gehaltene und inhaltlich immer sorgsam abwägende Darstellung. Das Vertiefungskapitel behandelt ausgewählte Formulierungsmuster in wissenschaftlichen Texten sowie Todes-/Traueranzeigen als formelhafte Texte. Das nun noch folgende Kapitel, das die (berechtigte) Frage nach dem Status von Textsorten und Textmustern als Konstruktionen stellt und eine mögliche Öffnung der Konstruktionsgrammatik auf die Textebene kritisch reflektiert, wäre besser (analog zu allen anderen Kapiteln) vor dem Vertiefungskapitel platziert.

Im Kapitel 6 werden gesprächsspezifische Routinen und Formeln, Paarsequenzen und Sequenzmuster, rituelle Muster (mit Bezug auf das Goffman'sche Face-Konzept) und ritualisierte Gesprächsphasen sowie kommunikative Gattungen als Muster auf Gesprächsebene besprochen. Auch hier wird sichtbar, wie unterschiedlich die Komplexität und der Abstraktionsgrad des Musterhaften ausfallen. An der Liste der musterhaften Einheiten zeigt sich noch etwas, die inhaltliche Struktur der Einführung betreffend: Das Kapitel zur Gesprächsebene wie auch die folgenden Kapitel (die hier aus Platzgründen knapper besprochen werden) zur Diskursebene, zu musterhaftem Sprachgebrauch im Rahmen des Spracherwerbs und in einzelnen Varietäten sowie zur sprachkritischen Perspektive auf Musterhaftigkeit liegen auf einer anderen Ebene als die vorherigen Kapitel. Denn all diese Kapitel bauen auf den vorangegangenen auf (sichtbar auch an den intertextuellen Verweisen) und berücksichtigen Musterhaftes auf (Mehr)Wort-, Satz- und Textebene. Hierunter fallen unter diskurslinguistischer Perspektive (Kap. 7) Argumentationsmuster/Topoi, Metaphern und Denkstereotype. Hervorzuheben unter den einzelnen Unterkapiteln - da sehr ausführlich und aufschlussreich - sind 
die exemplarischen Vertiefungen zu Topoi und Metaphern in Migrations- und in Wirtschaftskrisendiskursen sowie zu Denkstereotypen über Frauen im 19. Jhd. (sog. Backfische) und über Wessis und Ossis im 20. Jhd. Unter Spracherwerbsperspektive (Kap. 8) werden „das Ausmaß und die Rolle sprachlicher Muster beim ungesteuerten Spracherwerb theoretisch und im gesteuerten Spracherwerb sprachdidaktisch analysiert“ (S. 211), wobei (leider) nur system- und normgerechte Muster (s.o.) berücksichtigt werden. Hier wäre es interessant gewesen, gerade auch die irregulären Muster auf Wortbildungsebene zu betrachten (z.B. „falsche“ Verbbildungen wie abverreden statt verabreden, die man häufig bei Kindern beobachten kann). Unter varietätenlinguistischer Perspektive (Kap. 9) wird Musterhaftigkeit im Rahmen diachronischer, diakonzeptioneller, diatopischer, diastratischer sowie diaphasischer Variation behandelt, und die Autoren zeigen anhand von musterhaften Einheiten aus Dialekten und Soziolekten auf, dass sprachlichen Mustern zwar „stets eine charakteristische Dia-Markierung innewohnt, die jedoch für Veränderungen offen und zulässig ist“"(S. 264). Aus sprachkritischer Sicht (Kap. 10) halten sie fest, dass Musterhaftes, insbesondere auf (Mehr-)Wortebene („Floskeln“), durchaus Anlass zu sprachkritischer Beurteilung geben kann. Dabei repetieren sie den Topos, dass die laienlinguistische Sprachkritik den Sprachgebrauch, in diesem Fall die Verwendung musterhafter Mehrworteinheiten, pauschal und ohne die erforderliche Differenzierung und Reflexion abwertet, wohingegen die linguistisch begründete Sprachkritik (natürlich) „angesichts der laienlinguistischen Bewertungs- und Argumentationspraktiken [...] anderen Kriterien verpflichtet ist“ (S. 278) und Sprachgebrauch „grundsätzlich verwendungsbezogen [...] analysier[t]“ (S. 289). Diese Sichtweise ist weder neu noch lässt sich der laienlinguistischen Sprachkritik vorwerfen, gänzlich ohne Verwendungsbezug zu urteilen. Auf diese Gegenüberstellung hätte meines Erachtens verzichtet werden können.

Die Einführung schließt mit einem kurzen, vierseitigen Schlusskapitel, in dem die Frage erörtert wird, ob der Rekurs auf Musterhaftes „unkreativ“ (S. 291) sei. Die Frage verstehen die Autoren in dem Sinne, ob die Verwendung von sprachlich Musterhaftem im Sprachgebrauch unkreativ sei. Ihre Antwort fällt wie erwartet differenziert aus. Man müsse innerhalb des Musterhaften und je nach Verwendungskontext unterscheiden, zumal die endgültige Beurteilung bei den Rezipientinnen und Rezipienten liege: Was dem einen gefalle, müsse nicht auch der anderen gefallen. Die Frage lässt sich aber auch anders deuten, indem man den Rekurs auf Musterhaftes auf den Wissenschaftsdiskurs und die Frage nach der Kreativität auf das Thema des Buches bezieht: Ist eine Einführung zum Thema Musterhaftigkeit unkreativ? Hier fällt meine Antwort eindeutig aus: mitnichten. In der kurzen abschließenden Reflexion zeigen die Autoren nochmals auf, wie facettenreich und vielschichtig die Thematik ist, mit welchen Herausforderungen die 
Auseinandersetzung damit verbunden ist und welches Potential in der „Musterhaftigkeitsforschung“ liegt.

Das Buch ist in jeder Hinsicht eine Anregung, sich weiter mit dem Thema zu beschäftigen. Auf die leser*innenfreundliche Gestaltung auf inhaltlicher Ebene wurde eingangs schon hingewiesen. Darüber hinaus zeichnet sich die Einführung durch einen verständlichen und sehr gut lesbaren Stil aus. Gut gefällt nicht zuletzt das übersichtliche Layout. Im Fließtext sind wichtige Begriffe fett gedruckt, was die Orientierung erleichtert; wo es sich anbietet (z.B. bei Erläuterungen, Klassifizierungen) wird der Fließtext durch Aufzählungselemente unterbrochen; graphische Übersichten dienen dem Verständnis und der schnellen Nachvollziehbarkeit der Ausführungen. Auch wenn die Kapitel unterschiedliche Verfasser^innen haben, ist das Resultat ein stimmiges Ganzes. Angesichts der Vorzüge fallen die wenigen geäußerten Kritikpunkte nicht schwer ins Gewicht - bleibt nur, dem Buch viele Leser`innen zu wünschen.

\section{Literatur}

Bubenhofer, Noah. 2009. Sprachgebrauchsmuster. Korpuslinguistik als Methode der Diskursund Kulturanalyse. Berlin, New York: De Gruyter.

Feilke, Helmuth. 1996. Sprache als soziale Gestalt. Ausdruck, Prägung und die Ordnung der sprachlichen Typik. Frankfurt am Main: Suhrkamp.

Fix, Ulla. 1999. Textsorte - Textmuster - Textmustermischung. In: Marie-Hélène Pérennec (Hg.). Textlinguistik: An- und Aussichten. Lyon et al.: Institut d’Études Germaniques, 11-26.

Heinemann, Margot \& Wolfgang Heinemann. 2002. Grundlagen der Textlinguistik. Interaktion Text - Diskurs. Tübingen: Niemeyer.

Mangasser-Wahl, Martina (Hg.). 2000. Prototypentheorie in der Linguistik. Anwendungsbeispiele - Methodenreflexion - Perspektiven. Tübingen: Stauffenburg.

Steyer, Kathrin. 2013. Usuelle Wortverbindungen. Zentrale Muster des Sprachgebrauchs aus korpusanalytischer Sicht. Tübingen: Narr.

Steyer, Kathrin (Hg.). 2004. Wortverbindungen - mehr oder weniger fest. Berlin, Boston: De Gruyter. 